\title{
Impact of HIV and AIDS on the eldery: A case study of Chiladzulu district
}

\section{Anthony P. Sefasi \\ Catholic University of Malawi}

\section{Abstract}

HIV may affect the elderly in a number of ways. They may become infected themselves; their children may suffer prolonged illness and incapacity, and need the elderly to look after them; the same children may then die from the illness, leaving the elderly without the support of the next generation; this bereavement also leaves the older people to meet funeral costs and then to take care of orphans left behind. HIV has impacts on old people in ways that are social, economic, psychological and physical in nature.

\section{Aims}

We wanted to understand the socio-economic impacts of HIV/ AIDS on lives of older people following the death of their productive children, and to examine how the elderly struggle to take care of HIV/AIDS orphans. While it is generally appreciated that older people undergo such hardships, little research has been done in Malawi to unravel the magnitude of these problems. We hope that our findings will increase awareness and lead to more being done to alleviate the problems faced by older people as a result of HIV/AIDS.

\section{Results}

The results reveal that older people have no alternative but to provide care and support to their ill children, and following the death of their children to become parents again in taking care of the orphaned grandchildren. This is a particularly big challenge in a country like Malawi, dependent on hand-hoeing for subsistence agriculture, where food production requires people who are physically strong. $79 \%$ of older people taking care of HIV/AIDS patients or orphans were found to have limited or no information about HIV/AIDS. 31\% were dependent on relatives for support, while $69 \%$ sustained themselves through piece work, small business or farming.

\section{Conclusion}

Elderly people need more resources and assistance to enable them to fulfil their vital role in caring for HIV-AIDS orphans. They are also in critical need of more information about HIV-AIDS, as many are sexually active with multiple partners, and preventive information is currently not reaching this important section of the community.

\section{Introduction.}

According to the World Health Organisation and the United Nations AIDS Programme. 34.4 million people were living with HIV/AIDS as of the end of 2008 and AIDS had resulted in more than 25 million deaths since the first clinical evidence was reported in $1981^{1}$.

The high incidence of HIV/AIDS in the sub-Saharan African region is leading to a devastation of individual and family life. Studies in Eastern and Southern Africa indicate that women and children are AIDS' greatest victims ${ }^{2}$.

In Malawi according to a Ministry of Health 2007 report, about 970,000 people are living with HIV, with new infections estimated at 90,000 per annum. These figures pose an enormous challenge in terms of care for people who will soon develop AIDS, some of whom will die leaving orphans in need of support ${ }^{3}$.

Throughout sub-Saharan Africa, older people - particularly older women - are a key resource for combating AIDS and alleviating its impact ${ }^{3}$. At household level, they are the persons who provide daily care for both AIDS patients and children left orphaned by the pandemic. These older people have taken on new roles by providing care and financial support to orphaned children, playing child-rearing roles within their extended families and continuing their more traditional roles as advisors to their adult children and grandchildren. They largely absorb the enormous additional burdens placed on families and society by the AIDS pandemic.

According to a study in Zambia(1996) 57\% of primary care givers were grandparents. Of these, $37 \%$ were between 60 and 85 years of age. This 'skip-generation parenting' accounted for $45 \%$ of the cases in another study of 1996 in Zimbabwe. According to Kadzandira (2007) in countries that have a high prevalence of HIV and AIDS such as Malawi, older people are increasingly playing the principal role of caring for younger adults sick with HIV and AIDS and for orphaned grandchildren ${ }^{4}$.

The World Health Organisation (2002) reported that 30\% of all households in sub-Saharan Africa were headed by an older person. In Namibia, South Africa and Zimbabwe, $60 \%$ of orphans live with their grandparents. Up to $56 \%$ of carers for orphans and vulnerable children in Namibia are more than 60 years old 5 .

In terms of older people themselves being infected with HIV, UNAIDS (2005) reported that 2.8 million adults aged 50 years and older were living with HIV worldwide, representing $7 \%$ of all cases.

Since there have been few studies of this kind in Malawi, we investigated whether older people in Malawi - who account for $6 \%$ of the general population - are undergoing similar challenges in this era of HIV and AIDS.

\section{Objectives}

The main objective of the study was to establish the impact of HIV/AIDS on older people in Malawi using Chiradzulu district in southern Malawi as a case study.

\section{Methods.}

\section{Design}

We used both qualitative and quantitative approaches. Using in-depth interviews and focus group discussions, we collected data from 116 older people who were found to be taking care of HIV and AIDS orphans. We conducted the study between January and April 2010.

Questions and discussions during data collection centred on personal information, numbers of children, possible causes of death of the orphaned children's parents, the burden of care being undertaken, and mechanisms used to cope with this.

\section{Study population and Site}

This study was conducted in Chiradzulu district in the Southern part of Malawi, a district with a high HIV/AIDS prevalence. The age criterion for inclusion in the study was 60 years. (For individuals who did not know their age, we 
estimated it using major historic events - eg 'At what stage in your life did Dr. Kamuzu Banda first return to Malawi?')

The Group Village Headman and a child protection worker identified households where an older person was taking care of a terminally Ill HIV/AIDS patient or taking care of HIV/AIDS orphaned grandchildren. Once we knew the number of older people for possible inclusion per village we employed a simple random sampling technique to come up with our sample. Once an individual had consented to participate, s/he was interviewed using a questionnaire. Another sample was identified for focus group discussion, with 12 participants being drawn from different villages in the district.

\section{Results}

\section{Roles played by older people in caring for a sick} relative from within the household

$40 \%$ of the respondents had been guardians of a sick child or grandchild; while $38 \%$ reported having been providing financial and other support to the sick relative. $22 \%$ were recorded to have been providing palliative care and spiritual counselling to the patient prior to their death.

\section{Challenges faced by older people taking care of orphans.}

$59 \%$ of the sample reported that they had difficulty sourcing money for orphan grandchildren's school uniform, for food and for meeting hospital bills in case of illness. $22 \%$ of the sample indicated that taking care of orphaned grandchildren halted their own development because they were forced to be at home looking after the sick grandchild or taking the child to hospital. They particularly stressed that they had to spend their reserved resources to make the lives of their grandchildren better, while impoverishing themselves in the process. $19 \%$ of the respondents indicated that while they were able to support their orphaned grandchildren now, they were worried about what will happen to these children following their death; as to who will support the children, who will look after them when they are sick, and who will remind them to take their anti-retroviral treatment without fail every day.

\section{Challenges faced during illness and death of a family member.}

$43 \%$ of the respondents reported spending all the money they had on medication (which included both western and traditional) and on buying food. $44 \%$ of the respondents indicated having sold property to get money for meeting expenses both for medication and for the funeral ceremony. $12.5 \%$ indicated losing direct support from the deceased (who had been working and supporting them, financially and otherwise, before their illness made this impossible).

\section{Economic impact of HIV and AIDS on the Aged}

$55 \%$ of the enrolled elderly people were affected through death and sickness of their children who gave them support and care. One grandmother commented; "All the money had been used to care for my children. They all died and now I have the responsibility to look after the grandchildren." There was regret that an economically productive person had been lost by the household. Generally, the longer the illness dragged on, the more resources were depleted. For example one old lady recounted her ordeal as follows; "I cared for my daughter who suffered for three years at home. All the resources which she had were exhausted, and I started using my own resources thinking that she will get better." An old Lady of 77 years from Masikini Village lamented, "The illness and eventual death of my son exhausted all our financial resources and now I do not have anybody to ask for help and support."

\section{Factors that put older people at risk of getting infected with HIV.}

$56 \%$ of the respondents felt that lack of knowledge on HIV/ AIDS speeds up the infection rate among their age group, as most of the times preventive messages target the youth and not older people. One old lady explained that following the death of a young person, "we are asked to wash the dead body; most of the times we do not know the cause of death, bence we wash the body without gloves. This puts our lives at risk." $12.5 \%$ believed that taking care of a sick relative with HIV/AIDS without protection at home put them at risk of the infection. Respondents reported that having multiple sexual partners is common among older people.

\section{Discussion}

Old people face a lot of challenges because of the HIV/ AIDS pandemic. HIV/AIDS is wiping out productive children, and grandparents must assume responsibility for the orphaned grandchildren, without sufficient resources, and often without sufficient strength, to do so. These findings are consistent with studies worldwide from areas hardest hit by HIV/AIDS pandemic.

HIV/AIDS messages have not been reaching older people ${ }^{5}$. There is a great misconception that when people grow old they become sexually inactive. Our study established that in fact older people do commonly have multiple sexual partners, which is why we need to include them in our preventive messages on HIV/AIDS.

In a country like Malawi where there are no social pensions for older people, the HIV/AIDS impact is particularly intense. Some older people, as established by this study, have no alternative but to be beggars in an effort to make ends meet.

\section{Conclusion}

The impact of HIV/AIDS on older people in Malawi is diverse and deep. Social support networks are disrupted, and older people are left impoverished. Meanwhile they are also at risk of becoming infected themselves because preventive information on HIV/AIDS is not reaching them.

\section{Recommendation}

Diverse stakeholders should take an active role in alleviating the burdens being carried by older people in Malawi as a consequence of HIV/AIDS. Access to subsidized fertilizer and other amenities should not be age-based, as this leaves out older people who are taking care of orphans. Messages about the disease and its prevention strategies should not be limited to people in the reproductive age groups only, as is the case currently10. There is need for more research in this area for us to begin influencing policy change with Government.

\section{Acknowledgements.}

I would like to acknowledge support received from the following people; Marango Singini, Eluby Fukiza and Immaculate Sefasi without them this work would not have been possible. I would like to thank chiefs from the villages where participants were drawn, child protection workers and the participants themselves for their time and commitment to this exercise. 


\section{References}

1. World Health Organisation Community home-based care: Family care giving. Caring for family members with HIV/AIDS and other chronic illnesses: Impact on older women and girls. Geneva (WHO/ NMH/CCL/01.01) 2000.

2. World Health Organisation. Impact of AIDS on older people in Africa: Zimbabwe case study. Geneva, WHO. (http://whqlibdoc.who. int/hq/2002/WHO_NMH_NPH_ALC_02.12.pdf)

3. Chimwanza, A. and Watkins, S. Giving care to people with AIDS symptoms in sub-saharan Africa. AIDS Care.2004; 16 (7): 795-807.

4. Kadzandira J. Rural Aging and Livelihood challenges. Centre for Social Research. University of Malawi. Zomba.2007

5. Zablotsky, G. "Overlooked, ignored and forgotten: Older women at risk of HIV infection and AIDS." Research on aging 20: 460-775.

http://www.avert.org/older-people.htm. 1998

\section{WHO HEALTH STATISTICS ON Malawi}

This map is an approximation of actual country borders. Statistics

Total population $\quad \mathbf{1 3 , 5 7 1 , 0 0 0}$

Gross national income per capita (PPP international \$) 690

Life expectancy at birth m/f (years) $\quad 49 / 51$

Healthy life expectancy at birth $\mathbf{m} / \mathbf{f}$ (years, 2003) $\quad 35 / 35$

Probability of dying under five (per 1000 live births) 120

Probability of dying between 15 and 60 years $\mathrm{m} / \mathrm{f}$ (per 1000 population) $554 / 514$

Total expenditure on health per capita (Intl \$, 2006) 70

Total expenditure on health as \% of GDP (2006) 12.3

Figures are for 2006 unless indicated. Source: World Health Statistics 2008 